\title{
The Biophysical Function of the Human Eardrum
}

\author{
Janos Vincze, Gabriella Vincze-Tiszay \\ Health Human International Environment Foundation, Budapest, Hungary
}

\begin{abstract}
The hearing analyzer consists of two main systems: the peripheral hearing system, formed of the outer ear, the middle ear and the inner ear and the central hearing system, which contains the nervous pathways which ensure the transmission of the nervous influx and the hearing area where the information is analyzed and the hearing sensation is generated. The peripheral hearing system achieves the functions of transmission of the sound vibration, the analysis of the acoustic signal and the transformation of the acoustic signal in nervous inflow and the generation of the nervous response. The human hearing is characteristics: 1 . The eardrum vibrates from the sound waves; 2 . Auditory ossicles amplify the stimulus; 3 . In an oval window, the vibration is transmitted to the fluid space of the inner ear; 4. It vibrates the basilar membrane; 5 . What is pressed against the membrane tectoria; 6 . The stereocilliums of the hair cell bend, ion channels open; 7. Hair cell depolarizes; 8. Stimulus is dissipated in cerebrospinal fluid VIII (vestibulo $\neg$ cochlearis); 9. Temporal lobe primary auditory cortex (Brodman 41, 42); 10. Association pathways: speech comprehension (Wernicke area).
\end{abstract}

Keywords: Hearing analyzer, Hearing system, Human Eardrum.

\section{INTRODUCTION}

According to the frequency, the sounds classify as follows: infrasounds, $v<16 \mathrm{~Hz}$; proper sounds, $16 \mathrm{~Hz}<v$ $<20 \mathrm{kHz}$, ultrasounds, $v>20 \mathrm{kHz}$. The infrasounds and the ultrasounds cannot be perceived by the human ear [1].

The level of the minimum sound intensity which causes sounds perceivable by the human ear is called sensitivity threshold. The level of the maximum sound intensity for which the sounds do not produce painful effects, or temporary or permanent deafness is called painful sensation threshold. These two thresholds depend both on the sound frequency and the sound intensity. [2] The human ear perceives sounds with intensities ranging between 0 and $140 \mathrm{~dB}$. Table shows examples of sound intensity for various sound categories.

TABLE I. EXAMPLES OF SOUND INTENSITY FOR VARIOUS SOUND CATEGORIES

\begin{tabular}{|c|c|}
\hline Sound & L (dB) \\
\hline The leaves sre rusting & 10 \\
\hline Low-toned conversation & 30 \\
\hline Normal conversation & 65 \\
\hline Street noise & 90 \\
\hline Orchestral noise & 100 \\
\hline Engined aircraft (at $3 \mathrm{~m})$ & 120 \\
\hline
\end{tabular}

Examples of the values of the propagation of the sound in various environments are written in the next table.
TABLE II. EXAMPLES OF THE VALUES OF THE PROPAGATION OF THE SOUND IN VARIOUS ENVIRONMENTS

\begin{tabular}{|c|c|}
\hline Medium & $\begin{array}{c}\text { Velocity of sound } \\
(\mathbf{m} / \mathbf{s})\end{array}$ \\
\hline Air $\left(20^{0} \mathrm{C}\right)$ & 343 \\
\hline Water & 1435 \\
\hline Glycerine & 1750 \\
\hline Steel & 5100 \\
\hline Copper & 3400 \\
\hline Stone & 5000 \\
\hline Lead & 1300 \\
\hline Wood & $3000-4000$ \\
\hline Caoutchouc & 45 \\
\hline
\end{tabular}

\section{The MidDle EAR}

Through the auricle, the outer ear captures acoustic canals and transmits them as vibrations of the air molecules through the ear canal to the eardrum. The transmission of the outer ear canal is optimal for sounds with the frequency of $3500 \mathrm{~Hz}$ [3].

The middle ear achieves the transmission of the sounds through the solid environment. In order for the hearing signals to be transmitted to the internal ear through the middle ear, the transformations of the vibrations of the air molecules in the outer hearing canal in vibrations of the bones of the middle ear must be made so that the acoustic impedances of the two environments coincide. [4] This thing means that the middle ear realises an amplification of 
the sound captured by the outer ear, process which is achieved through two mechanisms:

a. the three articulate bones act like levers and the amplification factor is approximately $3 / 2$.

b. the ratio of the ear drum areas and the oval window, respectively is approximately 20:1, which means that for the same force, the resulting pressure which exerts on the oval window is 20 times higher.

The internal ear leads the sound to the liquid environment called lymph. The vibrations of the oval windows are transmitted to the lymph and through it they reach the level of the basilar membrane of the Corti organ. This way progressive waves are formed which propagate along the membrane. The displacements of the membrane cause extensions of compressions of the cillia, which determined the apparition of the nerve inflows at the level of the dendrites of the acoustic nerve which distributes to the lower pole of the hearing sensorial cells [5].

The exact mechanism through which the energy transported by the sound waves is transported by the sound waves is transformed in nervous inflow is not yet known. Several hypothesis have been issued about this mechanism. Hence, Helmholtz considers the localization of the high and, respectively, low frequency is different in the bone spiral, an idea confirmed by von Békésy (Nobel prize 1961). The microscopic analysis of the Corti organ certifies the fact that the threads of the ciliary membrane have different lengths according to the part of the spiral they are found on. The functioning of the system is based on the resonance of the vibrations on a well determined part of the basilar membrane and the effect on the ciliary cells. The sound wave causes the irritation of the ciliary cells which trigger the nervous inflow. G. von Békésy also determined that the waves which appear in the ear are not stationary, but mobile. The mobile waves of transversal movement on the basilary membrane, are formed, at low amplitude, at the level of the oval window and increase slowly, reaching maximum in a well determined point, after that they decrease rapidly, the shape of the mobile waves varies with frequency, the spatial position at which the amplitude is maximum depends on the frequency, moving towards the oval windowed when the frequency increases [6].

\section{EARDRUM}

The boundary between the outer and middle ears is formed by the eardrum, which is approx. $9 \mathrm{~mm}$ in diameter, slightly oval, moderately elastic plate. Its thickness is 0.1 $\mathrm{mm}$; its tensile strength is $100 \mathrm{kPa}$. It has a surface of 85 $\mathrm{mm} 2$, of which only $55 \mathrm{~mm} 2$ can be used for hearing. Its periphery forms a bony ring. Its outer surface is covered with skin in the continuation of the skin of the auditory canal, while the inside is covered by the mucosa of the tympanic cavity. The eardrum is like the diaphragm of a speaker. The eardrum is normally silver gray, shiny.
Between the two epithelial layers is a circular and radially running collagen fiber skeleton. The eardrum is inclined in both the craniocaudal and antero-posterior directions, making an angle of $55^{\circ}$ with the plane of the external auditory canal. The handle of the malleus is attached to its inner surface. On the eardrum, the handle of the malleus in the form of a white stripe causes a slight protrusion on the outer surface: stria mallearis. The upper, triangular part is weaker, translucent, the inner connective tissue layer is missing, while the others are denser parts. The two parts are separated by oblique wrinkles.

The eardrum can be divided into four quadrants: anterior-lower, anterior-upper, posterior-lower, and posterior-upper quadrants. The so-called light cone reflex can be seen on the anterior-lower quadrant, behind which the promontory is located in the tympanic cavity. This area is the usual site of paracentesis. Behind the anterior-upper quadrant is the opening of the Eustachian tube. Behind the posterior-bottom quadrant we find the round window. Behind the posterior-upper quadrant is the chorda tympani [7].

The eardrum is a two-dimensional surface and here its wave propagation can be characterized using Bessel functions. Bessel functions are particularly important for solving wave propagation problems and for static potential problems.

The Bessel differential equation is:

$$
x^{2} \frac{\partial^{2} y}{\partial^{2} x}+x \frac{\partial y}{\partial x}+\left(x^{2}-a^{2}\right) y=0
$$

; where: a-integer $0,1,2$.

The eardrum and auditory ossicles work perfectly when the air pressure outside the eardrum is the same as the air pressure in the tympanic cavity. The tympanic cavity pressure equalization is provided by the Eustachian tube, which presupposes good nose function. If nasal respiration is inhibited, e.g. enlarged nasal tonsil in children or nasal septal deviation and nasal polyps in adults nasal polyps, it can result in damage to the middle ear. As the eardrum on the Eustach tube travels with the upper part of the pharynx, it is how it is connected to the outside world. This is extremely important in the functioning of hearing and the development of diseases. The tympanic membrane and external air pressure are constantly equalized through the Eustach tube. When external air pressure pushes the eardrum into the tympanic cavity (retracted eardrum), the free movement of the ossicles bone chain is impeded due to compression. With good Eustach tube functioning, the tuba opens, and the tympanic pressure is equalized with each swallow. If no equalization is done, we will feel our ears are clogged.

The middle of the eardrum is retracted umbilically (umbo). The surface of the eardrum does not participate evenly in the vibrational movements. Békésy's studies shed 
light on the vibration conditions of the sub-surfaces of the eardrum. [8] The section between the umbo and the lower edge shows the largest angular deviation. The incudomalleolar joint is able to move about 5 degrees and thus follows the macroscopic movement of the eardrum due to any change in external pressure; at the same time, it is still capable of performing subtle movements in the service of prolific sound conduction. [9] At low frequencies, the entire eardrum moves; above $1500 \mathrm{~Hz}$, the manubrium of the malleus moves primarily. Sound vibrations are most effectively transmitted to the inner ear through the intact middle ear, which is air-guided hearing. Fig. 1.

The conical shape of the eardrum as well as its non-tight state is the most ideal for sound transmission. Own frequency: $1200-1800 \mathrm{~Hz}$, which corresponds to the frequency of the sounds to which the human ear is most sensitive. Due to the adhesion surface of the eardrum, it is a highly damped, linearly vibrating plate that transmits its vibrations to the malleus and thus to the auditory bone chain and to the air column in the middle ear.

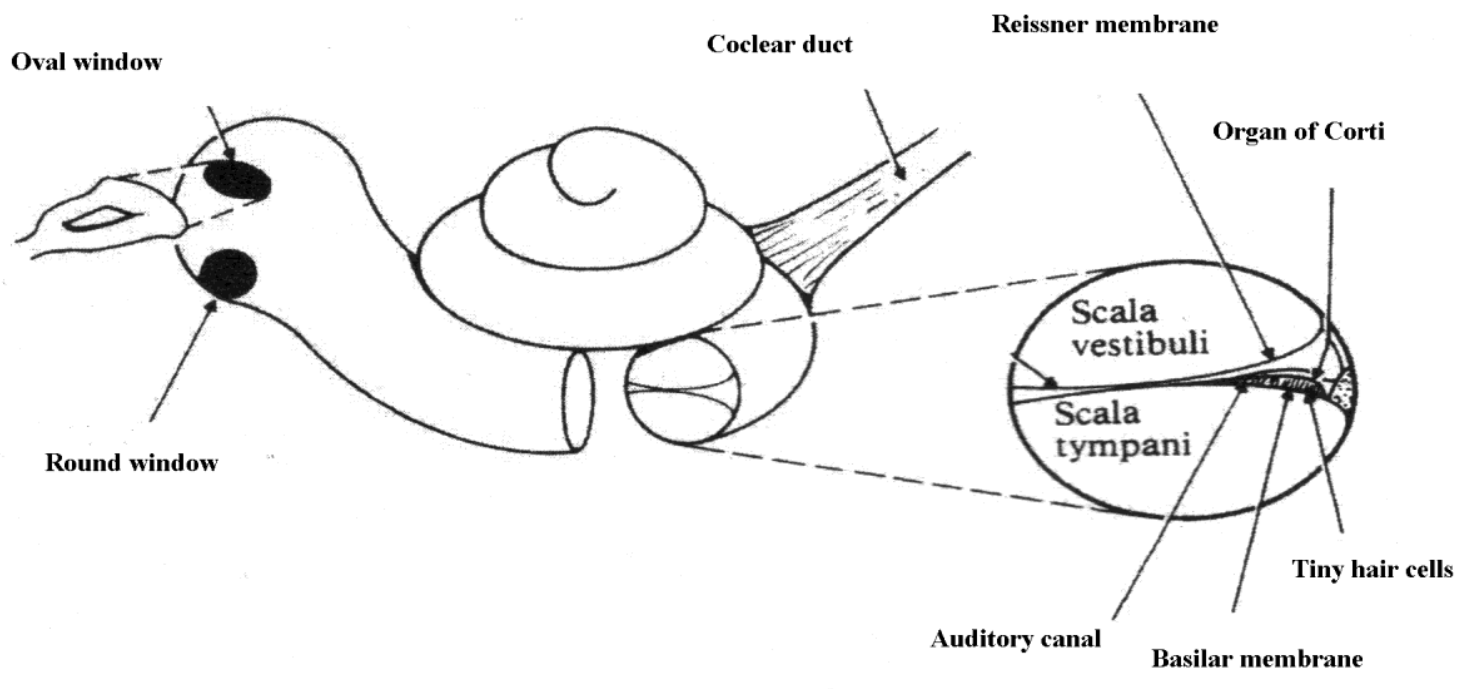

Figure 1. Connection of the middle ear to the outer and inner ears.

\section{AUDITORY OSSICLES}

There are three tiny bones in the tympanic cavity between the eardrum and the oval opening.

a. Malleus 6-8 $\mathrm{mm}$. The head (capitulum), the neck (collum) and projections can be distinguished on it. The strongest of the latter is the handle (manubrium), which has merged with the eardrum, causing stria mallearis on it.

b. Incus $7 \mathrm{~mm}$. A body and two protruding crura can be separated on it. The body forms a joint with the head of the malleus. Its short crus points backwards, its long crus runs parallel to the handle of the malleus, behind this formula. The lower part widens (processus lenticularis) and forms a joint with the head of the stapes.

c. Stapes $3 \times 3 \mathrm{~mm} 2$. On the third auditory bone, a head (capitulum), two crura and a base (basis) are distinguished. The head fits into the lenticular process of the incus and the base fits into the oval window.

\section{BIOPHYSICAL MODELING OF THE SOUND CONDUCTION OF THE AUDITORY OSSICLES}

It plays an acoustic role, transforming external sound vibrations, amplifying them and then transmitting them to the fluid system of the inner ear. It protects the inner ear from excessive sound effects [10].

The deflections of the eardrum are transferred to the base of the stapes by the lifting action of the auditory bone chain. Due to the fact that the functional surface of the eardrum is $55 \mathrm{~mm} 2$ and that of the stapes base is $3.2 \mathrm{~mm} 2$, the pressure at the base of the stapes is 18 times higher than that of the eardrum $(55: 3.2=17)$. This ratio corresponds to a $24.5 \mathrm{~dB}$ increase in sound pressure. To this must be added $2.2 \mathrm{~dB}$, since one crus of the auditory bone chain (one arm of the elevator) is one handle of the malleus, the other crus (the other arm of the elevator) is the long crus of the incus, 1.3 times longer than the handle of the malleus. Thus, the voice guidance system of the middle ear: It causes a pressure increase of $24.5+2.2=26.7 \mathrm{~dB}$. From all this we can conclude that the middle ear acts as a mechanical transformer [11]. 
The auditory ossicles can be considered as rigid bars and thus the sound waves propagate in a longitudinal form. In this case, the wave equation:

$$
\frac{\partial^{2} \Phi}{\partial t^{2}}=c^{2} \cdot \frac{\partial^{2} \Phi}{\partial x^{2}}
$$

The phase velocity c for longitudinal waves in a solid medium is given by the following formula:

$$
c=\sqrt{\frac{E}{\rho} \cdot \frac{1}{(3-6) \mu}}
$$

where: E - modulus of elasticity, $\rho$ - density, $\mu$ Poisson's ratio

With their reflex contraction, the m.stapedius and m.tensor tympani stiffen the auditory bone chain (70-80 $\mathrm{dB}$ sound above the hearing range) and reduce the amplitude of the system vibrations ( $10 \mathrm{~s}$ delay) - the reflex mainly reduces the transmission of deep sounds $-n$. facial paresis - causes hyperacusis. The stapes base moves around two axes [12]:

for weaker sounds - rotates around its transverse axis (Fig. 2.a);

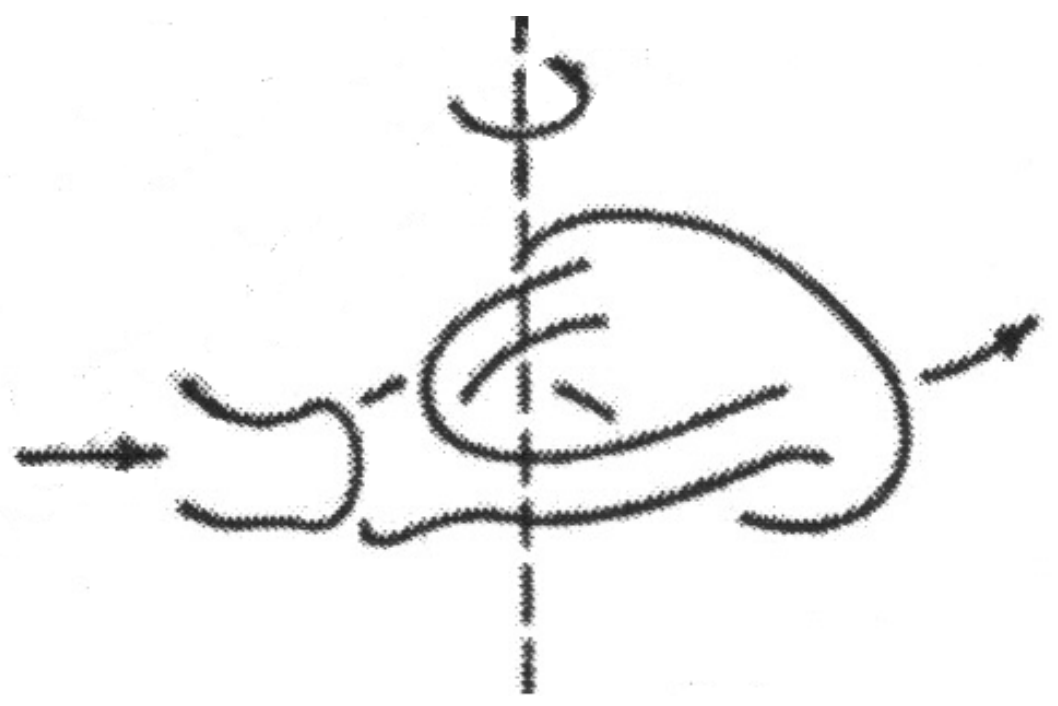

Figure 2. a. Rotates around its transverse axis.

In case of a strong sound - it moves around its longitudinal axis, then the amplitude of vibration is smaller (Fig. 2.b).

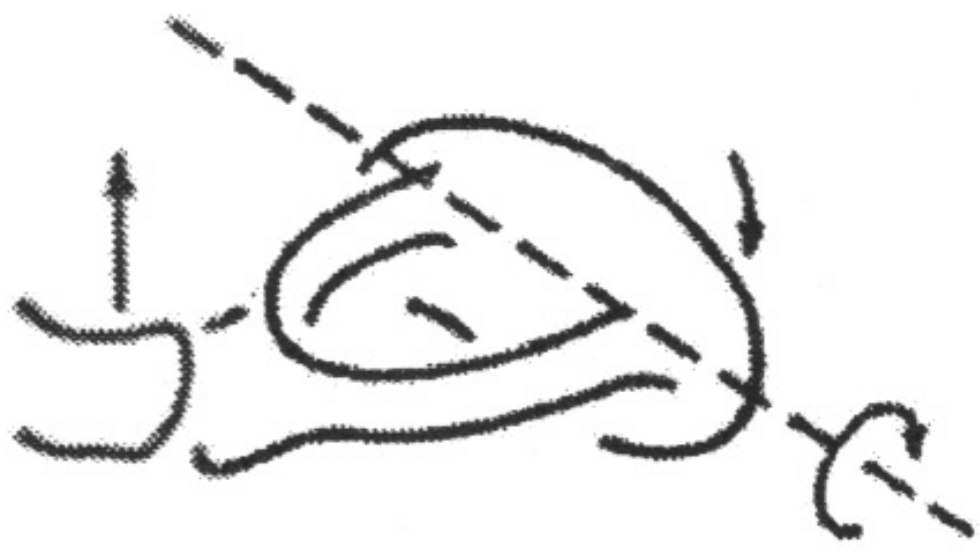

Figura 2. b. Moves around its longitudil axis. 
We have three (3) auditory ossicles because, with their spatial location, the middle ear is able to amplify weak sounds and at the same time is able to attenuate highintensity sounds. [13]

\section{BÉKÉSY'S PLACE THEORY (TONOTOPIC LOCALIZATION)}

The sound wave vibrates the basilar membrane only at the place corresponding to its frequency Oval window: tight basilar membrane - high tones. Tip of the cochlea: wide, loose basilar membrane - deep tones [14].

1. Sound stimulus creates a surface wave that extends from the oval window to the top of the cochlea.

2. Propagation rate decreases $(45 \mathrm{~m} / \mathrm{s}-2 \mathrm{~m} / \mathrm{s})$ with increasing membrane width and looseness

3. Phase - delayed waves catch up with the previous one, the amplitude increases and then suddenly decreases.

4. The location of the maximum of the envelope on the basilar membrane depends on the frequency of the sound, and its amplitude on the intensity.

5. Vibration of the basilar membrane displaces the hair cells of the organ of Corti, an electrical potential change begins.

6. The stimulus from a given location in the organ of Corti corresponds to the temporal lobe of the cerebral cortex.

7. delivers the information to its place: a feeling characteristic of the given sound is formed Strengthening role of external hair cells [15] Prestin voltage-sensitive motor protein in the membrane of external hair cells.

8. External hair cells detect displacement of the basilar membrane, $\mathrm{K}+$ channels open, depolarization.

9. Prestin: conformational change, longitudinal cell length change based on sound vibration - electromechanical transduction.

10. Vibrations of the basilar membrane are amplified.

\section{CONCLUSION}

The hearing analyzer consists of two main systems: the peripheral hearing system, formed of the outer ear, the middle ear and the inner ear and the central hearing system, which contains the nervous pathways which ensures the transmission of the nervous influx and the hearing area where the information is analyzed, and the hearing sensation is generated. The peripheral hearing system achieves the functions of transmission of the sound vibration, the analysis of the acoustic signal and the transformation of the acoustic signal in nervous inflow and the generation of the nervous response.

\section{REFERENCES}

[1] Vincze J. (1986). Medical Biophysics. Medical P. Budapest.

[2] Vincze J., Vincze-Tiszay G.: The Biophysical Modeling of the different Regulations in the Human Organism. Intern. J. Inovat. Studies Med. Sciences, 2020; 4(1):1-4.

[3] Vincze J. (1990). Biophysics. 1th Ed. NDP P. Budapest.

[4] Vincze J. (2015). Biophysics. 5th Ed. NDP P. Budapest.

[5] Berne R. M., Levy M. N. et all. (2014). Physiology. 7th Mosley, Elsevier.

[6] Gamow G., Cleveland J. M. (1997). Physics. Pretince-Hall, Inc. Englewood Cliffs, N. J.

[7] Russell K. H., Roth B. J. (2019). Intermediate Physics for Medicine and Biology. Elsevier.

[8] Békésy, Georg (1960): Experiments in Hearing. McGraw-Hill Book Company, New York.

[9] Vincze J. (2007). Interdisciplinarity, NDP P., Budapest.

[10] Vincze J. (2018). Medical Biophysics. NDP P., Budapest.

[11] Vincze J., Vincze-Tiszay G.: The Human Organism is a Biophysical-Biopsychological System. Technium, 2020; 2(7): 2935.

[12] Vincze J. (2008). Biophysics of the Phonation and of the Hearing. NDP P., Budapest.

[13] Békésy G.: Some Biophysical Experiments from Fifty Years Ago. Annu. Rev. Phsyiol. 1974; 36(1):1-18.

[14] Vincze J., Vincze-Tiszay G..: The Biophysical Adjustment in the Human Organism. J. Med. Res. Case Report, 2020; 2(3)1-7.

[15] Vincze J. (2021). BiophysicalVademecum. NDP P., Budapest. 